\title{
Spatial and configural factors in compound stimulus processing by pigeons
}

\author{
ROBERT G. COOK \\ Tufts University, Medford, Massachusetts \\ DONALD A. RILEY \\ University of California, Berkeley, California \\ and \\ MICHAEL F. BROWN \\ Villanova University, Villanova, Pennsylvania
}

\begin{abstract}
Three matching-to-sample experiments examined whether spatial or configural factors determined how the element arrangement of compound sample stimuli influenced matching accuracy in pigeons. Seven types of compound stimuli were tested. The arrangement of color and lineorientation elements in these compounds varied in terms of the spatial separation between the elements, the degree of consistency in element spatial location, and the number of bounded areas containing the elements. Matching accuracy was examined upon initial exposure to the compounds, during asymptotic conditions of shared attention, and with variation of sample durations ranging from .04 to $5.935 \mathrm{sec}$. In all three experiments, when spatial proximity, locational certainty, and the number of lines were precisely controlled or equated, no evidence for the proposed configural processing of "unified" compounds was found (Lamb \& Riley, 1981). Element spatial separation, and to a lesser degree perceptual limitations, determined compound performance. These results question our lab's previous evidence for configural compound processing by pigeons (Lamb, 1988; Lamb \& Riley, 1981). They suggest instead that pigeons independently and separately process the individual elements of color/line-orientation compounds, with element separation determining the distribution of processing between the elements.
\end{abstract}

Research on humans has shown that the organization of the elements forming a compound visual stimulus strongly influences its psychological properties (Ceraso, 1985; Garner, 1974, 1976). Riley and Leith (1976) similarly proposed that element organization may also influence how pigeons process compound stimuli. Since then, two reports from our lab (Lamb, 1988; Lamb \& Riley, 1981) have offered evidence in support of this proposal, obtained in variations of the element/compound delayed matchingto-sample (E/C DMTS) task.

In the E/C DMTS task, the pigeon is presented with either an element sample, consisting of one of two color elements (red or green, henceforth $\mathrm{R}$ or $\mathrm{G}$ ) or one of two line-orientation elements (vertical or horizontal, henceforth $\mathrm{V}$ or $\mathrm{H}$ ), or a compound sample, consisting of both a color and a line element (RH, RV, GH, GV). The sample is followed by a test in which the two values of a single dimension are pitted against one another (R vs. G or

This research was supported by National Science Foundation Grant BNS-7908839 to D. A. Riley. Additional support during the preparation of the manuscript was provided by National Science Foundation Grant BNS-525222 to R. G. Cook. We thank Sonja Yoerg, Evangeline Wheeler, and Cynthia Langley for their comments on earlier drafts. Request for reprints should be sent to Robert G. Cook, Department of Psychology, Tufts University, Medford, MA 02155.
V vs. H). The choice of the test stimulus that matches the sample or one of its dimensional components produces food reinforcement. Following element samples, the test stimuli are always from the dimension of the sample. Following compound samples, the pigeon is tested unpredictably with either set of dimensional tests, forcing stimulus control by both elements.

Using the E/C DMTS task, Lamb and Riley (1981) compared matching accuracy among several types of compounds. They found that the elements of a "unified" compound, consisting of colored lines in which both elements were in the same stimulus boundary, were matched more accurately than were those of "separated" compounds. The latter compounds consisted of a colored square separated from an achromatic line element by different distances. It was suggested that this compound matching difference was due to configural properties of the unified compound, which allowed it to be processed as a single unit and thereby reduced the effect of compound information load (Brown \& Morrison, 1990; Maki \& Leith, 1973; Riley \& Roitblat, 1978). In further testing of this idea, Lamb (1988) added a cuing procedure to the E/C DMTS task and found evidence suggesting that the elements of a spatially separated compound were processed independently of one another, whereas the elements of a unified compound were processed together, perhaps as a single unit. 
Both results suggested that pigeons may have different modes of processing compounds, depending on the compounds' configurations, with unified compounds being nonanalytically or configurally processed as single units and separated compounds being analytically or separately processed as two units.

Although the configural hypothesis is intriguing, aspects of each experiment leave the exact origins of these unified/ separated compound differences unresolved. Lamb's (1988) unified and separated compound stimuli differed not only in element configuration, but also in the spatial separation of their elements. The elements of his separated compound were separated by a $17-\mathrm{mm}$ gap not present in the unified compound. Hence his results may have been due to this difference in element spatial separation, and not to element configuration per se.

Lamb and Riley (1981) included the conditions necessary to isolate the influence of element separation from element configuration. Unfortunately, other confounds among their stimuli prevented any conclusion about the source of compound matching differences reported by them. First, two colored lines were used to form the unified compounds, whereas three lines formed the remaining compounds, element samples, and test stimuli. Second, this caused the separated compounds to form a square configuration, whereas the unified compounds were rectangular, providing an additional global cue to the latter compound's line orientation. Third, their unified compounds consistently appeared in a central location on the sample key, whereas the location of the color and line elements of their separated compounds varied unpredictably between trials. Such locational inconsistency retards second-order conditioning (Rescorla \& Cunningham, 1979), and we were concerned that it might have influences in the matching context too. Finally, analyses presented below will suggest that Lamb and Riley's (1981) experiments may not have been conducted under conditions of shared attention-that is, conditions in which more than a single element was being processed from the compound samples (Kraemer \& Roberts, 1987; Riley \& Roitblat, 1978).

Because Lamb's (1988) experiment confounded element configuration with element spatial separation, and because Lamb and Riley's (1981) experiment confounded element configuration with other complicating and less interesting factors, these important initial explorations leave the original question unanswered. Do pigeons process compounds both configurally and analytically, and if so, how do the configuration of the elements and their degree of spatial separation influence these processes?

In the present experiments, we tested an unconfounded set of compound stimuli in order to answer the preceding questions. The strategy was twofold and similar to that of Lamb and Riley (1981). The first part was a parametric manipulation of element spatial separation to properly isolate configural properties from element proximity effects. This was done with four separated compounds made from identical color and line elements, but separated by $2,6,10$, or $14 \mathrm{~mm}$.

The second part of the strategy was to examine a set of compounds that differed in element configuration, but whose elements were spatially close together. Three compounds of this type were tested and were similar in design to those of Lamb (1988). In the unified compound, both elements were contained in a single colored form. In the close compound, the color and line elements were adjacent to one another, but did not overlap. In the superimposed compound, the color and line elements were superimposed. Figure 1 shows the four element stimuli used in these experiments, and Figure 2 shows examples of the different compound stimuli.

These compounds thus formed two general classes. One consisted of the four spatially separated compounds, whose elements were identical, but which differed in separation, and the second consisted of the four spatially adjacent or proximal compounds (unified, superimposed, close, $2 \mathrm{~mm}$ ), whose elements were spatially contiguous, but which differed in configuration. The status of the 2-mm separated compound was considered equivocal, so this compound was included in both groups. It was designed as one endpoint of our parametric spatial manipulation, but its elements were either close together $(2 \mathrm{~mm}$ vertical) or superimposed ( $2 \mathrm{~mm}$ horizontal; see Figure 2).

As suggested above, the locational predictability or (un)certainty of each element dimension may also have been an important factor in Lamb and Riley's (1981) experiments. We chose to manipulate this factor rather than eliminate it, as was done for the differences in the number
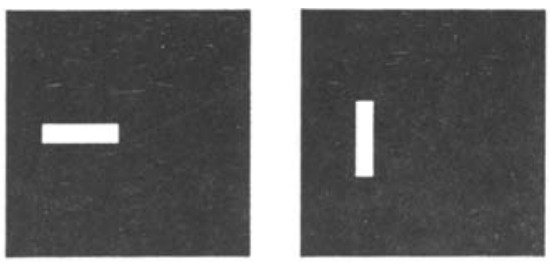

Horizontal

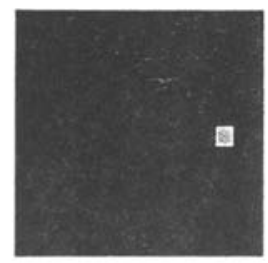

Red

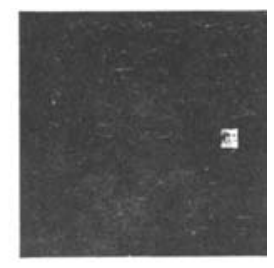

Green

Figure 1. Examples of the element stimuli used in these experiments. 


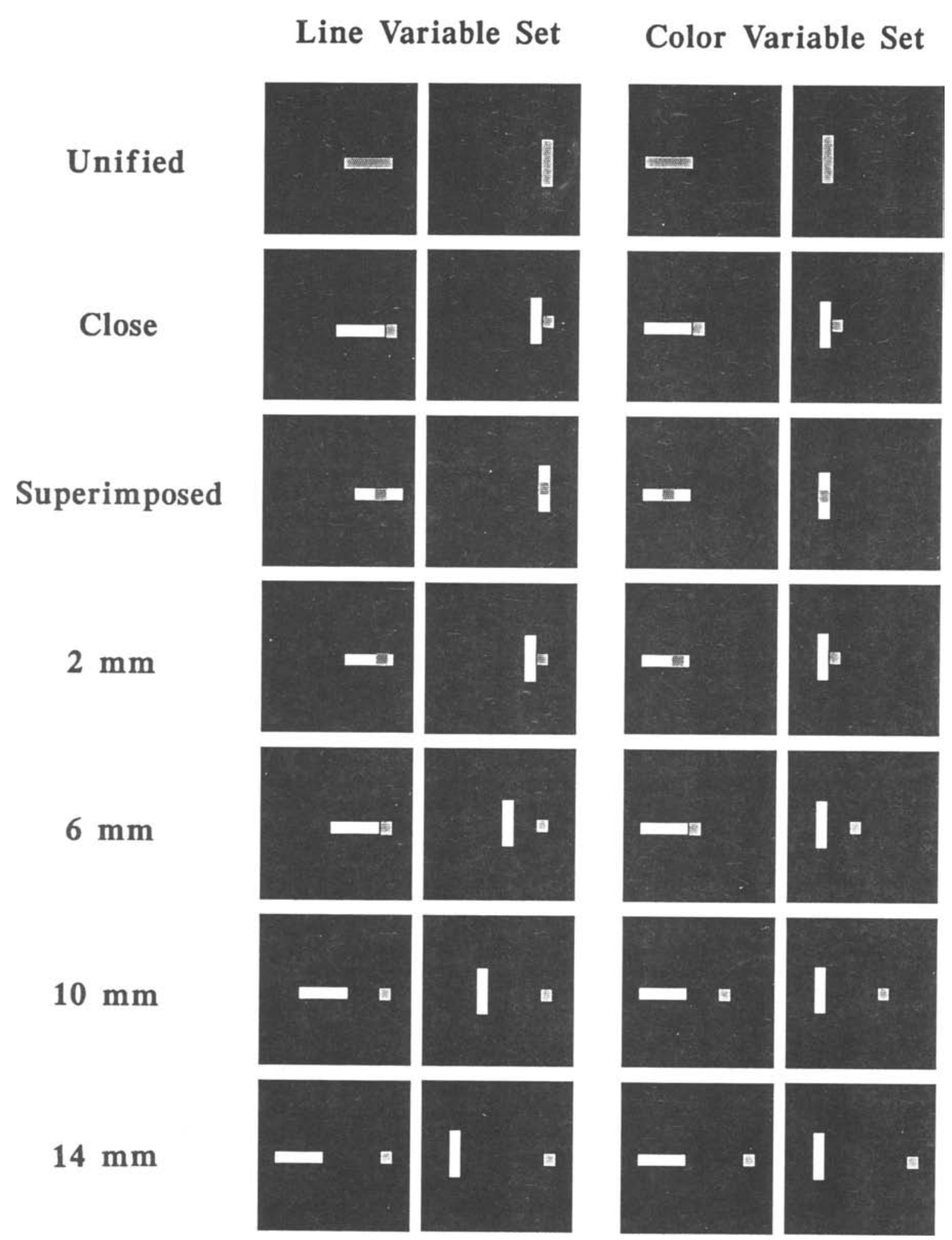

Figure 2. Examples of the line- and color-variable compound stimuli used in these experiments. Examples of the vertical and horizontal samples are shown in combination with only one of the two colors used. 
of lines and the global orientation cue, through the use of the same-shaped single line in all seven compounds. This manipulation involved affixing the response key location of one of the sample elements and then varying the distance of the second element between different compounds. Three birds were tested with a set of stimuli in which the line element varied in location between compounds, while the color element remained in the same location (see Figure 2, line-variable set). Two other birds were tested with the color element in variable key locations, while the location of the line element was fixed (see Figure 2, color-variable set).

Performance with these seven compound types (unified, close, superimposed, $2 \mathrm{~mm}, 6 \mathrm{~mm}, 10 \mathrm{~mm}$, and $14 \mathrm{~mm}$ ) was then examined in the E/C DMTS task. Three testing conditions were investigated. These were chosen because they were thought to be the most sensitive to any processing differences between the compounds. Experiment 1 examined performance upon initial exposure to the compounds. Experiment 2 examined performance after asymptotic and stable conditions of shared attention were clearly established. Experiment 3 examined compound performance at different sample durations.

\section{EXPERIMENT 1}

This experiment examined the transfer of element matching ability to the compound stimuli. The objective was to investigate compound stimulus processing early in testing, prior to any effects of experience that might alter compound processing (Brown \& Morrison, 1990; Grant \& MacDonald, 1986). Although not previously examined, performance early in testing may provide especially important data for evaluating element-organization effects. For example, if the unified compounds were processed in a configural manner, new matching rules for this compound type might have to be learned (Grant \& MacDonald, 1986), resulting in lower initial levels of accuracy with this compound.

\section{Method}

Subjects. The subjects were 5 pigeons (Columba livia) of mixed breed. Two were experimentally naive; 3 had learned a $V$ versus $\mathrm{H}$ line discrimination presented with in-line projectors 6 months before these experiments. All were maintained at $75 \%$ of their freefeeding weights during testing and had free access to water and grit.

Apparatus. Testing was conducted in a three-key operant chamber $(31 \times 36 \times 38 \mathrm{~cm})$ with black walls. The front wall had three pecking keys (BRS/LVE Model 121-16) $23.4 \mathrm{~cm}$ above the floor. The center key was square $(3.3 \times 3.3 \mathrm{~cm})$, and the side keys were round ( $3.3 \mathrm{~cm}$ in diameter). The side keys were $10.4 \mathrm{~cm}$ from the center key. The food hopper, which was $15.6 \mathrm{~cm}$ below the center key, contained mixed grain. The stimuli were rear-projected onto the keys by slide projectors (Kodak Model E-2). Stimulus onset and offset were controlled by high-speed shutters (Uniblitz 26LOA3X5 for the side keys and 214LOA0T5H for the center key). Experimental events were controlled by a PDP-11/23 computer (Digital Equipment Corp.) in conjunction with locally constructed interfacing equipment.

Stimulus materials. Stimuli were composed from the color and/or line-orientation dimensions. Color elements were $2 \times 2 \mathrm{~mm}$ red (Kodak Wratten filter No. 16) or green (No. 58) squares. Line- orientation elements were single $2 \times 10 \mathrm{~mm}$ vertical or horizontal white lines.

Two sets of compound stimuli were formed by combining one value from each dimension. In the line-variable set (LV set), the color element always appeared in the same pecking key location ( $7 \mathrm{~mm}$ from its center to the right edge of the key), while the center of the line element was located $0,2,6,10$, or $14 \mathrm{~mm}$ to the left of the center of the color element. In the color-variable set (CV set), the location of the line elements was fixed ( $7 \mathrm{~mm}$ from its center to the left edge), while the colored element varied in location in a similar manner. Each stimulus set included the following compound types.

Unified compounds. These compounds appeared as a colored line $(2 \times 10 \mathrm{~mm})$ on either the right (LV set) or the left (CV set) side of the key.

Superimposed compounds. These compounds appeared as a white line element with the $2 \times 2 \mathrm{~mm}$ colored square superimposed in the center of the line $(0 \mathrm{~mm})$ on either the right ( $\mathrm{LV} \mathrm{set}$ ) or the left (CV set) side of the key.

Separated compounds. Four different compounds were constructed by varying the spatial separation $(2,6,10$, or $14 \mathrm{~mm})$ between the center of the color element and the center of the line element.

Close compounds. These compounds consisted of spatially adjacent color and line elements that did not overlap. Close compound performance was derived from each session's performance with the 2-mm compound vertical samples (GV, RV) and the 6-mm compound horizontal samples (GH, RH). The close compound was not tested in its own 16-trial block as were the six other compounds (see the procedural description below). The reason for this was to maintain the procedural integrity of presenting the four separated compounds in different blocks, while equating the number of presentations for specific RV, GV, RH, and GH element combinations between the compound types. An additional separate block of close compound samples would have meant extra repetitions of stimuli that were the same as the $2-\mathrm{mm}$ vertical and 6-mm horizontal samples in each session. Because of this conflict and our compromise solution, the results for the close and 2-mm and close and 6-mm compounds are not entirely independent. As a result, omnibus analyses of variance (ANOVAs) comparing these specific compound conditions were not conducted, except in one case.

Procedure. The birds were autoshaped to peck the keys. The autoshaping trials consisted of a 5-sec presentation of the white warning stimulus on the center key, followed by a $2.5-\mathrm{sec}$ presentation of one of the two line-element samples. The center key was then turned off and the matching test stimulus was presented for $5 \mathrm{sec}$ on one of the two side keys (randomly selected). This was followed by presentation of the food hopper for $1.8 \mathrm{sec}$. A peck to the warning stimulus or the test stimuli advanced the trial to the next component, but the procedure was gradually changed to always require a response. When consistent responding was obtained, line-element MTS trials were formed by adding the incorrect test stimuli and making food available only after correct choices.

Each MTS trial began with presentation of the warning signal on the center key. A peck to the warning signal resulted in its replacement with the sample stimulus. The sample was presented for a specific duration or fixed number of pecks as described below. Immediately after sample termination, the test stimuli were presented on the side keys for 5 sec or until a choice was made. A peck to the matching test stimulus produced 1.8 -sec access to the food hopper, whereas a peck to the nonmatching stimulus caused the test stimuli to remain on until the 5-sec test interval expired. All intertrial intervals were $10 \mathrm{sec}$. Trials without a choice were presented again.

Following autoshaping with the line elements, 10 sessions composed of 80 line-element $(40 \mathrm{~V} / 40 \mathrm{H})$ zero-delay MTS trials were conducted. At this point, 40 color-element autoshaping trials were added. Upon consistent responding, color-element MTS trials were 
introduced in the same way as before. MTS training with $\mathbf{4 0}$ colorand 40 line-element trials continued for 10 sessions. At this point, a fixed-ratio schedule requiring 15 pecks (FR15) to the sample replaced the 2.5-sec sample duration used up to that point. Beginning with Session 88, each bird's FR requirement was reduced until accuracy was consistently between $80 \%$ and $90 \%$ correct with each element dimension (Bird 56, FR9; Bird 57, FR3; Bird 69, FR3; Bird 25, FR3; Bird 53, FR7).

Sessions including compound samples were then introduced. A peck to the test element that matched an element in the compound sample produced $1.8 \mathrm{sec}$ of access to food; a peck to the test stimulus not in the sample caused the stimuli to remain on until the 5-sec test interval expired. Each session with compound samples consisted of seven 16-trial blocks, with each block testing a different sample type. The testing order of these seven sample-type blocks was randomized for each session. For the 16-trial element block, each element ( $R, G, V$, and $H)$ was presented as a sample four times, with the correct test stimulus counterbalanced between the left and right keys. For the six 16-trial compound blocks, each combination of elements (RV, RH, GV, and $\mathrm{GH}$ ) was presented four times, with each dimension (color or line orientation) being tested on two trials, with the correct test stimulus counterbalanced between the left and right side keys. The order of these 16 trials was randomized within each of the seven blocks in each session. Eight additional element "warm-up" trials started each session. These were not analyzed. Hence, 120 total E/C DMTS trials were presented in a daily session. Compound transfer testing lasted 10 sessions.

\section{Results}

Two questions guided our analysis. The first concerned how much information was processed from each compound during its presentation. This was measured by overall matching accuracy (the mean of color- and line-test accuracy) for each compound. Because of Lamb and Riley's (1981) and Lamb's (1988) results, we were primarily interested in comparing performance among compound types, especially with the unified compound.

Our second question was concerned with how the properties of the component elements influenced accuracy. For example, compound accuracy can be divided into performance on the color and line tests. Alternatively, compound accuracy can be analyzed according to an element's degree of locational certainty - that is, according to whether it was the variable or the fixed element of its stimulus set.

The influence of these properties was examined with different ANOVAs that grouped the results accordingly. Analyses for the effect of dimension of test compared color- and line-test results. Analyses for the effect of locational certainty compared the fixed-element (color tests for Birds 56, 69, and 53 and line tests for Birds 57 and 25 ) and variable-element results (line tests for Birds 56, 69 , and 53 and color tests for Birds 57 and 25).

Overall accuracy. Figure 3 displays the mean accuracy for the element and the seven compound types during the 10 transfer sessions. During the 1st transfer session, mean compound accuracy (76.4\%) was significantly above chance [50\%; single mean $t$ test, $t(4)=8.5, p<.05$ ]. Choice accuracy did not change over the 10 transfer sessions, however; neither the main effect for sessions $[F(9,280)<1]$ nor the interaction with sample type $[F(54,280)<1]$ was significant in a two-way ANOVA

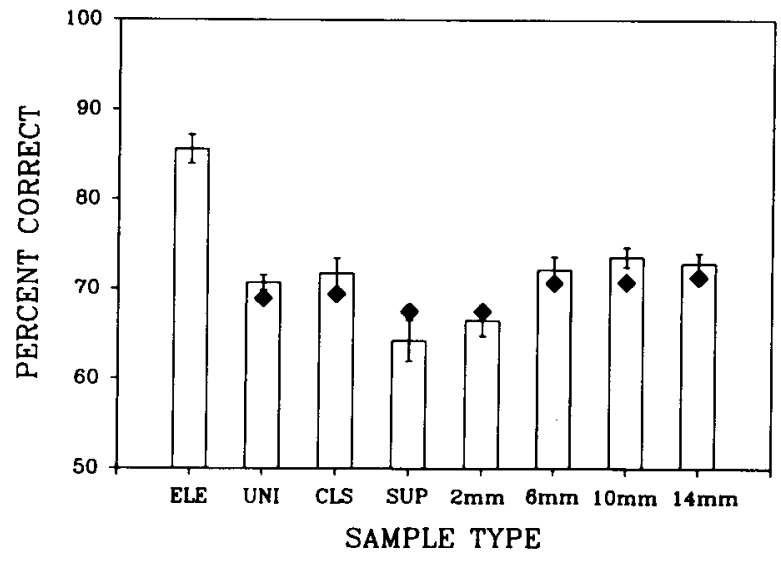

Figure 3. Mean percentage correct with the element and seven compound sample types in Experiment 1. The diamonds represent predicted overall accuracy from a distributed single-element model of compound processing described in the main text and in the Appendix. Error hars represent the SEM. ELE = element; UNI = unified; CLS = close; SUP = superimposed.

(sample type $\times$ sessions). Results from the 10 transfer sessions were thus combined in subsequent analyses.

In agreement with many earlier findings, element matching accuracy $(85.6 \%)$ was significantly higher than compound accuracy $[70.03 \% ; F(1,4)=128.6, p<.001$; one-way repeated measures ANOVA]. Of more interest were the differences in accuracy between the compound conditions $[F(5,20)=8.8, p<.001$; one-way repeated measures ANOVA]. ANOVAs comparing the compound types in a pairwise manner found the following pattern: unified $=$ close $=6 \mathrm{~mm}=10 \mathrm{~mm}=14 \mathrm{~mm}>$ superimposed $=2 \mathrm{~mm}$ [based on repeated measures ANOVAs with a critical $F$ value of $F(1,4)>7.7, p<.05$ ].

Analyses of element properties. Each bird's mean accuracy on color and line tests for the eight sample types is displayed in Figure 4. It shows that each bird was strongly influenced by element separation, with increasing element separation from 2 to $14 \mathrm{~mm}$ causing test accuracy for the two dimensions to diverge.

Analyses of the four separated compounds $(2,6,10$, and $14 \mathrm{~mm}$ ) with the planned ANOVAs for dimension of test and locational certainty revealed no significant interactions between either of these properties and separated compound type [compound type $x$ dimension of test, $F(3,12)<1$; compound type $\times$ locational certainty, $F(3,12)<1]$. Apparently, neither dimension of test nor locational certainty had a consistent influence on the matching of the elements in the separated compounds.

Nevertheless, each bird clearly showed a large asymmetry in its matching of color and line elements as a function of separation, with Birds 57, 69, and 56 matching line elements better than color elements and Birds 53 and 25 matching color elements better than line elements for the widely separated compounds. Therefore a third, and post hoc, analysis was done. This ANOVA (compound type $\times$ dimensional preference) reclassified the results 

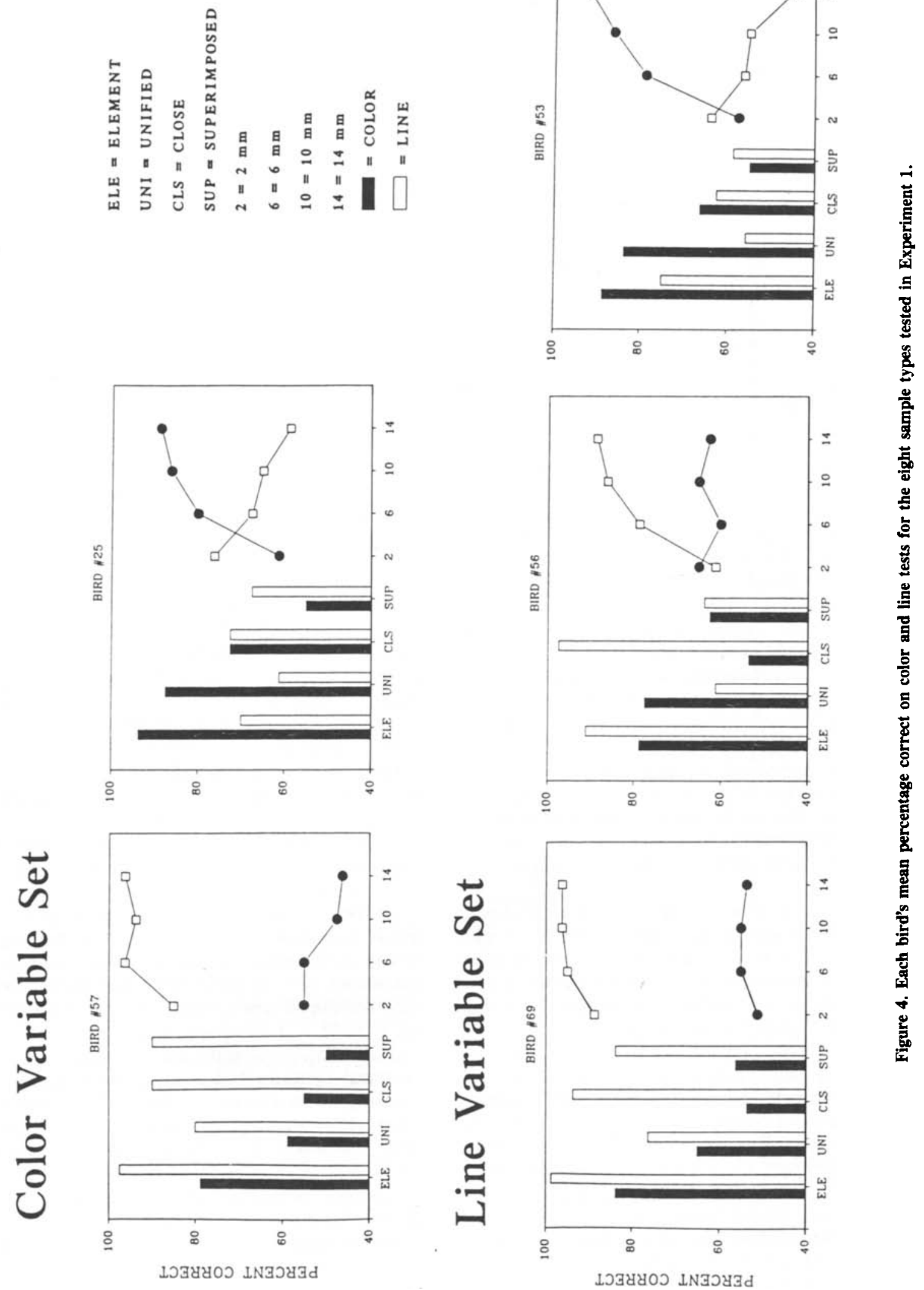


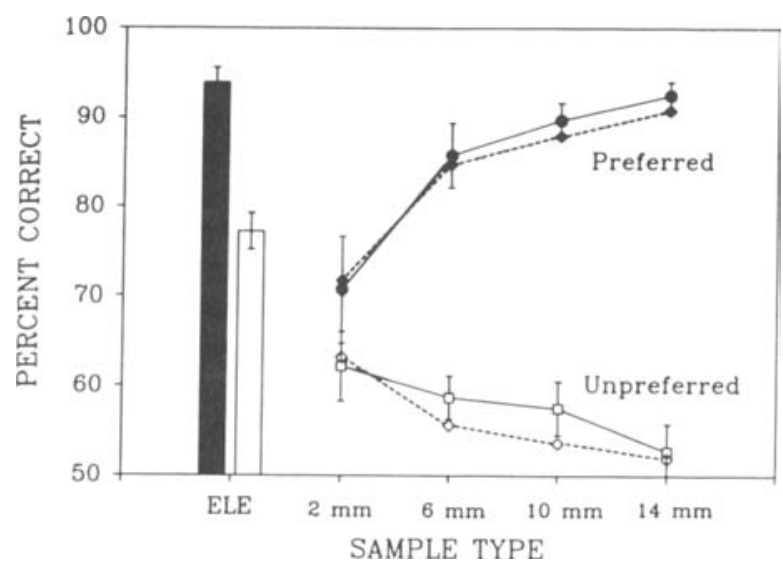

Figure 5. Mean percentage correct as a function of dimensional preference for the element and separated compounds during Experiment 1. Preferred and unpreferred labels denote the combination of each bird's most and lenst accurate dimension. The values for the element conditions were computed to be in accord with this classification of the compound performance. The diamonds, connected by the dashed lines, depict the predicted performance from the distributed single-element model. Error bars represent the SEM.

according to each bird's preferred and unpreferred dimension (defined as the dimension with the highest mean accuracy across sample types) and revealed a significant interaction between separated compound type and dimensional preference $[F(3,12)=12.5, p<.001]$. Figure 5 displays this interaction between preference and element separation. As separation increased, accuracy on tests of the preferred dimension significantly increased $[F(3,12)$ $=16.8, p<.001]$, whereas accuracy on tests of the unpreferred dimension significantly decreased $[F(3,12)=$ $3.8, p<.05]$. Thus, the difference in matching accuracy on the preferred and unpreferred dimensions was inversely related to element separation.

Additional analyses of only the 14-mm separated compound further suggested that only the preferred element was being processed. This was indicated by the equivalent levels of accuracy between tests of the preferred dimension after the 14-mm compound $(92.5 \%)$ and tests of the preferred dimension as an element sample [93.9\%; difference $t$ test, $t(4)=.85$ ]. Correspondingly, test accuracy with the 14-mm unpreferred element was at chance [52.7\%; single mean $t$ test, $t(4)=.94$ ].

Analyses of the four proximal compounds (unified, close, superimposed, and $2 \mathrm{~mm}$ ) revealed a pattern different from that found with the separated compounds. The repeated measures ANOVAs of dimension of test, locational certainty, and dimensional preference revealed that the only significant interaction occurred between compound type and dimension of test $[F(3,12)=6.3, p<.01]$. There were no significant interactions between proximal compound type and locational certainty $[F(3,12)<1]$ or compound type and dimensional preference $[F(3,12)<1]$. Note that this analysis and the corresponding ones of Ex- periments 2 and 3 were the only violations of our procedure of not comparing close and 2-mm compound performance within an ANOVA involving other compounds.

This interaction between proximal compound type and color- and line-test accuracy is shown in Figure 6. Wilcoxon rank-sum tests of color- and line-test accuracy revealed no reliable differences with the unified compound, whereas line matching accuracy was significantly higher with the superimposed compound $(p<.05)$ and marginally higher with the close and 2-mm compounds $(p=$ .10).

\section{Discussion}

Experiment 1 revealed little difference in overall accuracy among the seven compound types. The unified configuration of color and line in a single form conferred no processing advantage over the close, $6-\mathrm{mm}, 10-\mathrm{mm}$, or 14-mm separated compounds. Apparently, when the number of component lines is precisely controlled, when confounding global cues are removed, and when differences in element locational certainty are equated, the facilitation in matching accuracy with unified compounds reported by Lamb and Riley (1981) is not found. Accuracy was virtually identical with both the spatially comparable unified $(70.7 \%)$ and the close $(71.7 \%)$ compounds.

We believe that the reduced accuracy with the superimposed and 2-mm compounds was due to the perceptual masking or degradation of the color element during sample presentations (see also Farthing, Wagner, Gilmour, \& Waxman, 1977). Recall that these conditions are alike in that all four superimposed stimuli (RV, RH, GV, $\mathrm{GH})$ and two of the 2-mm stimuli (RH, GH) have the color element residing within the brighter white line element, perhaps making it more difficult to perceive and process. In both cases, color matching was very poor $(56.8 \%)$.

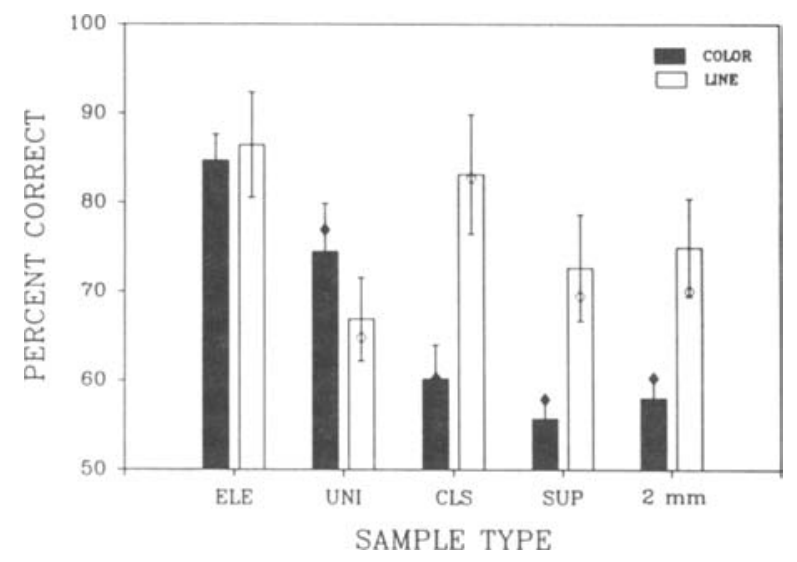

Figure 6. Mean percentage correct for color and line tests for element and proximal compound sample types during Experiment 1. The diamonds depict predicted performance from the distributed single-element model. Error bars represent the SEM. ELE = element; UNI = unified; CLS = close; SUP = superimposed. 
Further evidence in support of this explanation is presented in the later experiments.

Although element separation had no influence on the overall amount of information processed from each compound, it had large effects on the distribution of processing to the individual elements. When elements were close together, test accuracy was determined by the dimensional attributes of color and line. But as element separation was increased, a growing asymmetry in the matching of the two component elements emerged and appeared to be determined by individual preferences of the birds ( 2 of the 3 line-experienced birds preferred the line dimension).

This large matching asymmetry for the elements of the separated compounds is very similar to findings in two previous reports by Kraemer and Roberts $(1985,1987)$. They found that with visual/auditory compounds (Kraemer \& Roberts, 1985) and houselight/line-orientation compounds (Kraemer \& Roberts, 1987), only the italicized elements were processed. This restricted processing of the compound's elements produced element-level matching accuracy on tests of the processed element and chance responding on tests of the unprocessed element. The same pattern was found with the 14-mm compound in the present experiment.

Kraemer and Roberts (1987) accounted for this with a restricted-processing explanation, in which pigeons limit their processing to the same element either for strategic reasons or as a hard-wired preference. The restrictedprocessing hypothesis is an example of a class of compound processing theories which assume that only a single element is encoded from any compound stimulus. Singleelement hypotheses have been proposed before as explanations of E/C DMTS results with color/line-orientation compounds and rejected (Riley \& Roitblat, 1978) because compound accuracy typically exceeds $75 \%$. The latter value represents the maximum possible accuracy for such models (given perfect accuracy on tests of the processed element $[100 \%]$ and chance responding on tests of the unprocessed element [50\%].

The similarity of our separated compound results to those of Kraemer and Roberts $(1985,1987)$ suggested that examination of a single-element model might nevertheless be fruitful. Because the restricted-processing hypothesis assumes that the same component is always processed, it cannot account for the above-chance matching of both elements observed in some of our compounds. To adjust for this, we developed a single-element model which would allow the processing of a compound's elements to be more flexibly distributed, but which retained the essential assumption that only a single element or its informational equivalent was processed per compound trial. Below, we show that both Experiment 1 and Lamb and Riley's (1981) data can be successfully described with such a distributed single-element model.

In our model, compound accuracy was determined by two types of trials: (1) trials in which the dimensional information relevant to the test was "attended to" (Riley \& Leith, 1976) or " activated by" (Grant \& MacDonald,
1986) the compound sample, and (2) trials in which the relevant information was not available. Accuracy for processed trials was estimated from performance on element trials, and accuracy on unprocessed trials was assumed to be at chance $(50 \%)$. Compound accuracy was predicted by finding the best-fitting distribution of processed and unprocessed trials. A more detailed description of the model may be found in the Appendix.

The model returns two theoretically important values relevant to compound processing. The first is a predicted value for overall compound performance, given actual element performance and the restriction that only one element's worth of information is processed per trial. The second value is an estimate of the total number of processed elements per compound trial needed to account for the observed levels of matching accuracy (generated by eliminating the single-element restriction on the model; see the Appendix).

The values predicted by the model under the singleelement assumption are displayed as diamonds in Figures 3,5 , and 6. There was an excellent fit between the predicted and obtained values for overall compound accuracy $\left[\chi^{2}(6)=.49, p>.05\right]$, as well for the element tests of the separated compounds $\left[\chi^{2}(7)=.73, p>.05\right]$ and proximal compounds $\left[\chi^{2}(7)=.67, p>.05\right]$. Thus our pigeons' performance can be well described by assuming that only a single "element" of information was processed per compound trial. This conclusion is further amplified by the values generated from the unrestricted model, which has an additional free parameter and generates the best possible fit, but which nevertheless needed only 1.06 processed elements per compound trial to account for mean compound accuracy. It should be noted that the model is silent about whether this limited processing was distributed between both elements within a trial, as might be suggested by the shared-attention hypothesis (Maki \& Leith, 1973), or whether it was alternately distributed to one or the other element across trials, as might be suggested by the restricted-processing hypothesis (Kraemer \& Roberts, 1985).

In summary, no evidence was found for configural compound processing. The overall amount of information processed for each compound was the same regardless of configuration, except for two cases in which perceptual degradation of the component elements seemed to be a factor. When evaluated with a distributed single-element model, the amount of processed information was estimated to be equivalent to one element per trial, with the distribution of this limited processing between the elements influenced by the spatial separation of the elements. Processing was restricted to the "preferred" element when the two were far apart, but it was increasingly shared between the elements as they were brought closer together.

\section{EXPERIMENT 2}

Lamb and Riley (1981) argued that the benefits of configural compound processing should be most evident un- 
der conditions of shared attention or high information load because of the higher rate at which information from unified elements can be encoded. Our modeling of Experiment 1 suggested that only one element per compound trial was being processed. This implies that our compounds were not being tested under conditions of shared attention.

The success of the model prompted us to reexamine Lamb and Riley's (1981) results. Simulations with our unrestricted model found that one processed element per trial was all that was needed to describe compound accuracy in their first experiment also. For their compound types, these values were: unified, 1.19; superimposed, .9; close, 1.05; far, .93. This analysis suggests that Lamb and Riley's experiments were also not conducted under conditions of shared attention.

As a result, no clear test examining element configuration and element spatial separation effects under this theoretically important condition had yet been conducted. Because of this, we continued to train and test our pigeons in the expectation that with experience they would begin processing information from both element dimensions during compound trials.

\section{Method}

Subjects and Apparatus. The subjects and apparatus were the same as those used in Experiment 1.

Procedure. The E/C DMTS procedure of Experiment 1 continued to be used, except that the sample FR was changed to 20 pecks for all birds. The birds were tested for three 20-session blocks. Over these three blocks, mean compound matching accuracy increased from $77.6 \%$ to $84.1 \%$ to $86.0 \%$. During the third 20 -session block, daily accuracy appeared to be stable and asymptotic. An ANOVA (5-session blocks $\times$ sample type $\times$ dimension of test) dividing the last 20 sessions into four 5-session blocks revealed no reliable main effect of blocks or interaction of blocks with sample type. Because compound accuracy was not increasing and was significantly above the maximum single-element value $[75 \% ; t(4)=7.4, p<.05]$, only data from these last 20 sessions were analyzed.

\section{Results}

Overall accuracy. Analyses were again guided by the two questions discussed earlier. How much information was processed from each compound? Which element properties influenced performance? Displayed in Figure 7 is the mean overall accuracy for the element and seven compound stimulus types. Element matching accuracy (97.1\%) was again significantly higher than matching accuracy with the compound samples $[86.0 \% ; F(1,4)=$ $76.8, p<.001]$. Information from both elements was now being encoded during compound samples. This condition of shared attention was indicated by the fact that compound accuracy was significantly above $75 \%$ and by the extremely poor description of the results by the distributed single-element model $\left[\chi^{2}(6)=19.53, p<.05\right.$; the diamonds in Figure 7 are the predicted values for Experiment 2]. The unrestricted version of the model further supports this conclusion, with values of $1.2,1.46$,

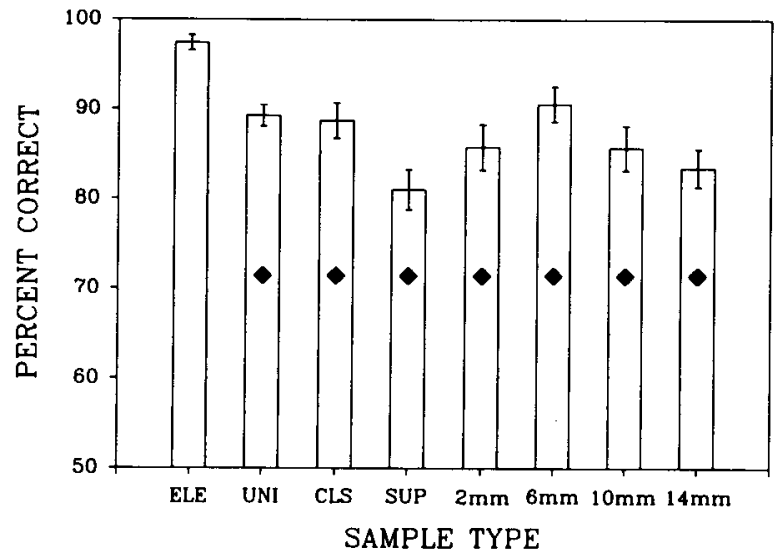

Figure 7. Mean percentage correct for the element and seven compound sample types. The diamonds are the predicted overall performance from a distributed single-element model. Error bars represent the SEM. ELE = element; UNI = unified; CLS = close; SUP $=$ superimposed.

and 1.52 processed elements per compound trial during the three 20 -session blocks, respectively.

Despite the increased accuracy and documented conditions of shared attention, the pattern of accuracy among the compound types was not different from the pattern in Experiment 1 (see Figure 7). There was again a significant difference in overall accuracy among the compound types $[F(5,20)=4.2 p<.01]$, but as in Experiment 1 , this continued to be due to the superimposed compound. Because comparisons of accuracy between the unified and six other compounds were of the most theoretical interest, six repeated measures ANOVAs were conducted; they revealed the following pattern: unified = close, $2 \mathrm{~mm}, 6 \mathrm{~mm}, 10 \mathrm{~mm}, 14 \mathrm{~mm}>$ superimposed [critical $F$ value in these ANOVAs, $F(1,4)>7.7$, $p<.05]$.

Analyses of element properties. Because Experiment 1 suggested that the controlling properties for the separated compounds (dimensional preference) and proximal compounds (element dimension) were different, separate analyses were continued. Figure 8 displays each bird's mean accuracy on color and line tests with each sample type. Comparison with Figure 3 reveals an important change from Experiment 1.

The preferred dimension for Birds 57 and 53 changed during Experiment 2. When tested after separated compounds, Bird 57 now matched line tests more accurately than color, whereas Bird 53 matched color tests more accurately than line. Table 1 documents the time course of these changes. Comparison with accompanying element sample accuracy shows that this change was specifically associated with compound performance and not dimensional matching accuracy in general.

With this reversal, all 5 birds now more accurately matched the locationally variable elements of their respective stimulus sets. That is, birds trained with the color- 

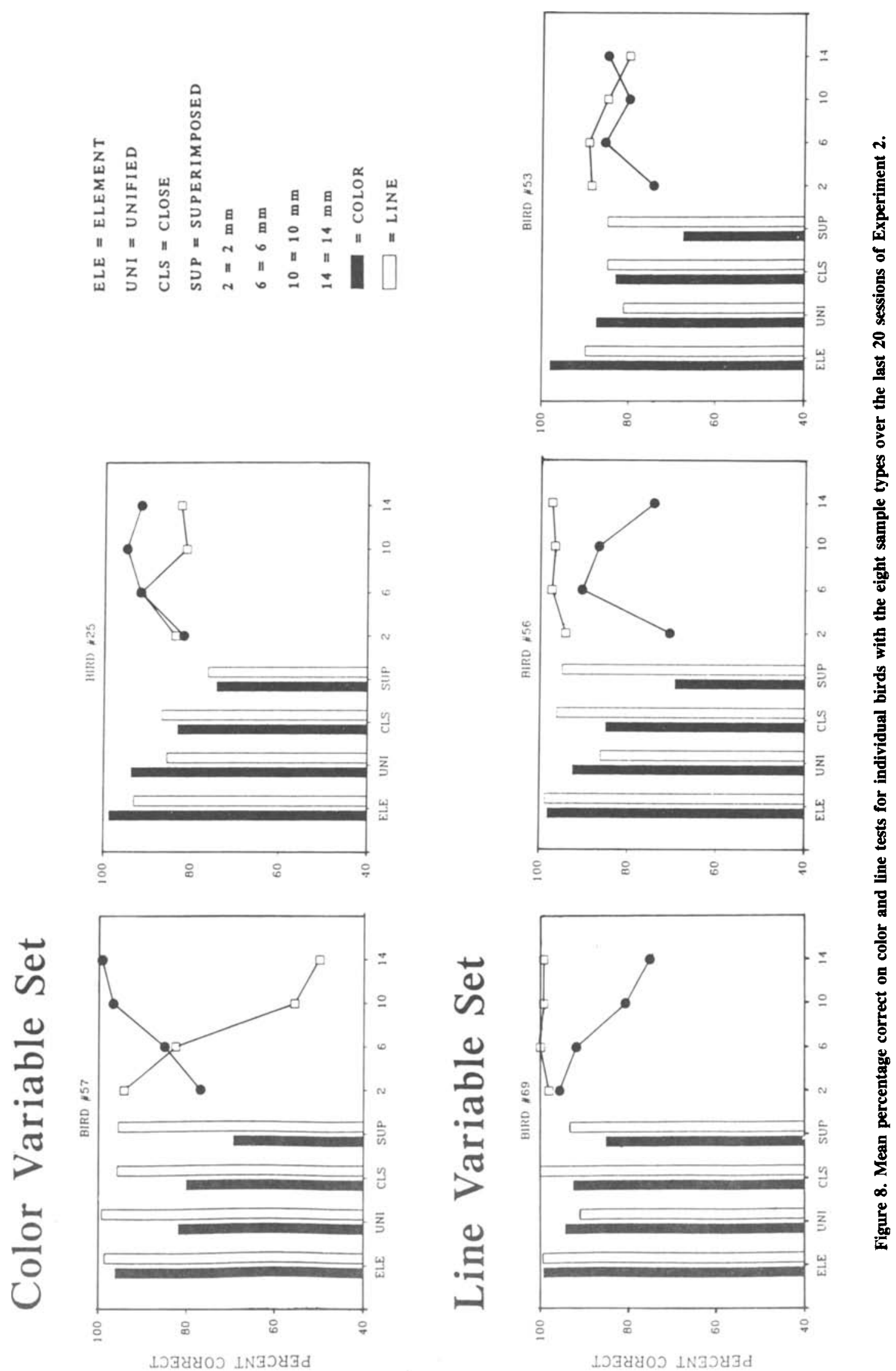
Table 1

Percentage Correct for Birds 57 and 53 for Different Sample Types Across the 60 Sessions of Experiment 2

\begin{tabular}{|c|c|c|c|c|}
\hline \multirow[b]{2}{*}{ Sessions } & \multicolumn{2}{|c|}{ Element } & \multicolumn{2}{|c|}{$\begin{array}{c}10-\mathrm{mm}+14-\mathrm{mm} \\
\text { Separated Compounds }\end{array}$} \\
\hline & Color & Line & Color & Line \\
\hline \multicolumn{5}{|c|}{ Bird 57} \\
\hline Transfer & 78.7 & 97.5 & 46.8 & 95.0 \\
\hline $1-5$ & 87.5 & 92.5 & 70.0 & 70.0 \\
\hline $6-10$ & 87.5 & 92.5 & 83.2 & 59.7 \\
\hline $11-15$ & 92.5 & 100 & 91.3 & 55.0 \\
\hline $16-20$ & 95.0 & 100 & 93.7 & 50.0 \\
\hline $21-40$ & 96.8 & 98.1 & 93.4 & 53.4 \\
\hline $41-60$ & 96.2 & 98.7 & 98.1 & 52.8 \\
\hline \multicolumn{5}{|c|}{ Bird 53} \\
\hline Transfer & 88.7 & 75.0 & 89.3 & 50.9 \\
\hline $1-5$ & 97.5 & 85.0 & 97.5 & 46.2 \\
\hline $6-10$ & 100 & 80.0 & 96.2 & 48.7 \\
\hline $11-15$ & 97.5 & 87.5 & 97.5 & 55.0 \\
\hline $16-20$ & 92.5 & 70.0 & 96.2 & 54.0 \\
\hline $21-40$ & 95.0 & 78.7 & 89.6 & 73.4 \\
\hline $41-60$ & 98.1 & 90.0 & 82.5 & 82.5 \\
\hline
\end{tabular}

variable set were more accurate on color tests, whereas birds trained with the line-variable set were more accurate on line tests. For the four separated compounds, ANOVAs identical to those in Experiment 1 revealed a main effect of locational certainty $[F(1,4)=16.2, p<.02]$ and no effects of dimension of test. As element separation increased from 6 to $14 \mathrm{~mm}$, accuracy on tests of the fixed element significantly decreased $[F(2,8)=7.0, p<.01]$, while accuracy on tests of the variable element did not change $[F(2,8)<1]$. Thus, as displayed in Figure 9, the asymmetry in element matching as a function of element separation observed in Experiment 1 continued-except that this effect was now governed by locational certainty rather than dimensional preference.

For the proximal compounds, dimensional properties of color and line continued to be more important (see Figure 10). There was a significant interaction between proximal compound type and dimension of test $[F(3,12)=$ $7.5, p<.01]$. Wilcoxon rank-sum tests comparing colorand line-test accuracy for each compound revealed no reliable difference with the unified compound, whereas line matching accuracy was significantly higher in the close, superimposed, and 2-mm compounds.

Additional analyses were conducted with the proximal compounds to further examine the role of perceptual degradation in the matching of superimposed compounds. These analyses examined the influence of line-element proximity and placement on color-element matching. If perceptual degradation were an important factor, color matching should improve with increasing distance from the brighter line element. A repeated measures ANOVA comparing color-test accuracy among the superimposed, 2-mm, and 6-mm compounds revealed that color matching significantly increased with growing distance from the line element [superimposed, $73.1 \% ; 2 \mathrm{~mm}, 79.8 \% ; 6 \mathrm{~mm}$, $89.0 \% ; F(2,8)=16.8, p<.01]$. Color-element matching also seemed to depend on its position within a com- pound. Color matching was significantly better when the color element was located just outside of the line element than when it was located at the center of the line element, as revealed by the significantly higher level of color matching in the close as opposed to the superimposed compounds $[F(1,4)=42.8, p<.001]$.

\section{Discussion}

With additional experience, the pigeons encoded information from both elements of the compound samples during Experiment 2. As argued by Lamb and Riley (1981), the benefits of configural processing should be most evident under such shared-attention conditions. Yet although the information load per compound trial was greater than it was in Experiment 1 (1.52 as opposed to 1 element per trial), no differences in matching accuracy between the unified and other compounds emerged that were attributable to configural processing. Accuracy was essentially identical with unified $(89.3 \%)$ and close $(88.8 \%)$ com-

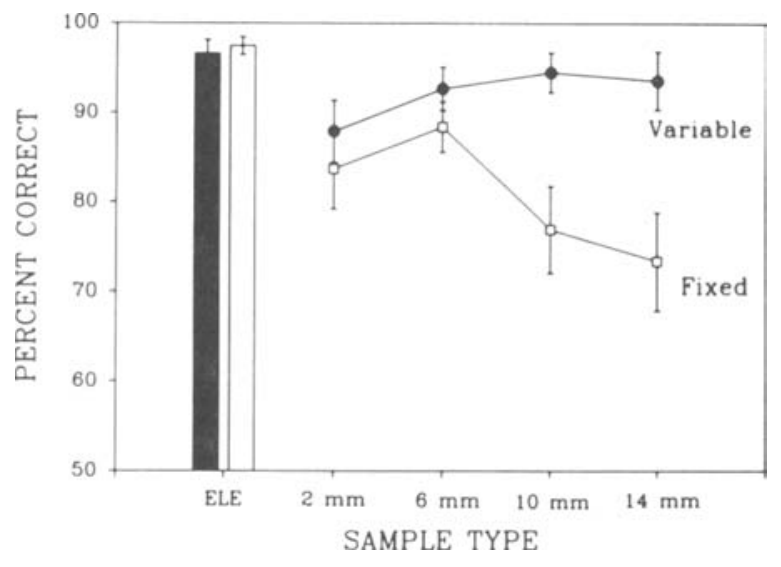

Figure 9. Mean percentage correct as a function of the variable/ fixed classification for the element and separated compound types during Experiment 2. Error bars represent the SEM.

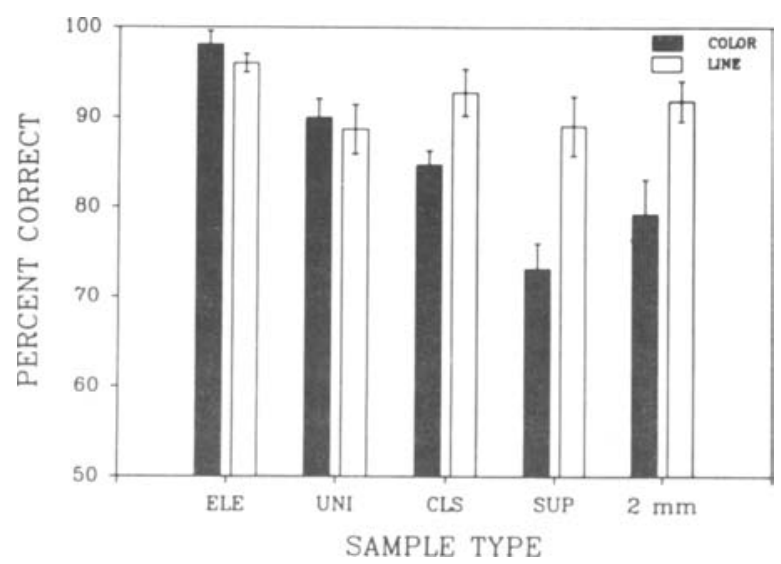

Figure 10. Mean percentage correct for color and line tests for element and proximal compound sample types in Experiment 2. Error bars represent the $S E M$. ELE = element; UNI = unified; CLS $=$ close; SUP $=$ superimposed. 
pound samples, in spite of the dissimilar physical arrangement of the component elements.

The poorer accuracy with the superimposed compounds continued to be best explained by the perceptual masking of the component elements, with the same mechanism probably responsible for the slightly reduced accuracy observed for the 2-mm compound also. Both conditions again supported the lowest levels of matching accuracy on color tests in comparison with the other compounds, although the accuracy had improved considerably over that observed in Experiment 1.

The most material change relative to Experiment 1 was the supplanting of dimensional preference by locational certainty as the determining factor in the element-selection effects observed for the separated compounds. In Experiment 2, all birds processed the variable elements of their respective stimulus sets more effectively. The explanation for this intriguing and unexpected result is not clear. In other situations, animals prefer conditions in which information is provided about upcoming events, even if these events are unavoidable and aversive (e.g., Miller, Marlin, \& Berk, 1977). Because one of our element dimensions was consistently in the same location, the identification and encoding of the remaining inconsistently placed element may have reduced situational uncertainty in an analogous manner.

In summary, despite the documented condition of shared attention and its accompanying increase in information load relative to Experiment 1, Experiment 2 also yielded no evidence for configural compound processing through element unification. With the exception of a limited perceptual degradation effect, the overall amount of information processed from each compound was the same, regardless of its configuration.

\section{EXPERIMENT 3}

Experiment 3 examined compound matching as a function of the temporal duration of the sample. This was done for two reasons. The first was to compare matching accuracy with the different compounds at short, controlled sample durations. This was of interest because the processing benefits of element unification might have been masked in Experiment 2 by the use of the FR sample requirement. If the birds compensated for the less efficient encoding rates of nonunified compounds by taking longer to complete the requirement, accuracy with these compounds might have risen to levels equivalent to the still more efficiently encoded unified compounds. If this was occurring, any shortening of the sample's duration should decrease accuracy with the nonunified compounds more than it would decrease accuracy with the unified compound. The higher rate at which information can be configurally encoded per unit time should allow the unified compound to better withstand decreases in the sample's duration.

The second reason for carrying out Experiment 3 was to compare element and compound performance at long sample durations. One of the clearest predictions of the shared-attention explanation of the matching difference between element and compound samples is that given sufficient encoding time this difference should disappear (Maki \& Leith, 1973; Riley \& Roitblat, 1978). The reasoning behind this is similar to that presented above. In this case, when given enough time, the animal should have the opportunity to process both elements of any compound and overcome the capacity limitation suggested to exist when two elements have to be processed instead of one. This prediction has not fared well in previous experimental tests, with element matching accuracy remaining higher than compound matching regardless of the duration of the sample (Brown \& Morrison, 1990; Lamb \& Riley, 1981; Santi, Grossi, \& Gibson, 1982). We tested this prediction again.

Six sample durations, ranging from .04 to $5.935 \mathrm{sec}$, were used with the same stimuli and procedure as in the two previous experiments. These sample duration values were identical to those tested by Lamb and Riley (1981).

\section{Method}

Subjects and Apparatus. These were the same as in Experiment 2.

Procedure. The only difference between the present procedure and the daily E/C DMTS procedures described earlier was that in each session the birds were tested with a different sample duration. There were six sample durations: $.04, .11, .295, .805,2.185$, and $5.935 \mathrm{sec}$. Each duration was tested once in a six-session block. The order of testing the six durations was randomized within a block. Six of these six-session blocks were conducted.

\section{Results}

Overall accuracy. Displayed in Figure 11 is the overall accuracy with the element and compound samples for each sample duration. Accuracy was equal in the two conditions up to $.805 \mathrm{sec}$, at which point compound matching dropped off and remained consistently below element matching. A repeated measures ANOVA (sample type [element vs. compound mean] $\times$ sample duration) showed

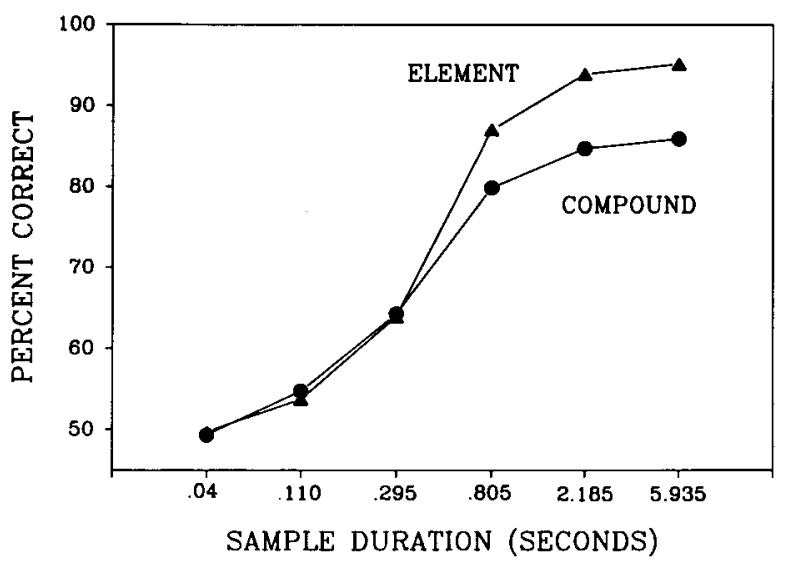

Figure 11. Mean percentage correct for the element and the combined compound conditions for the six sample durations tested in Experiment 3. For purposes of comparison, the spacing of the sample duration values is the same as in Lamb and Riley (1981). 
this sample type $\times$ sample duration interaction to be significant $[F(5,20)=3.6, p<.02]$.

Because the main purpose of the experiment was to look for unified compound matching superiority as a function of sample duration, only statistical comparisons of the unified condition with the other compound conditions were conducted. Three repeated measures ANOVAs (compound type $X$ sample duration) revealed no significant compound type main effects or compound type $x$ sample duration interactions for overall accuracy between the following compounds: unified $=$ close, $6 \mathrm{~mm}, 10 \mathrm{~mm}$. Significant compound type main effects or interactions with sample duration were found between the unified and superimposed $[F(5,20)=3.51, p<.02]$, unified and $2-\mathrm{mm}$ $[F(1,4)=16.2, p<.02]$, and unified and 14-mm $[F(1,4)$ $=24.7, p<.001]$ compounds: unified $>$ superimposed, $2 \mathrm{~mm}, 14 \mathrm{~mm}$. The top panel of Figure 12 shows the sample duration curves for the unified and the three compounds found not to significantly differ, while the bottom panel shows the sample duration functions for the unified and the three compounds found to be different.
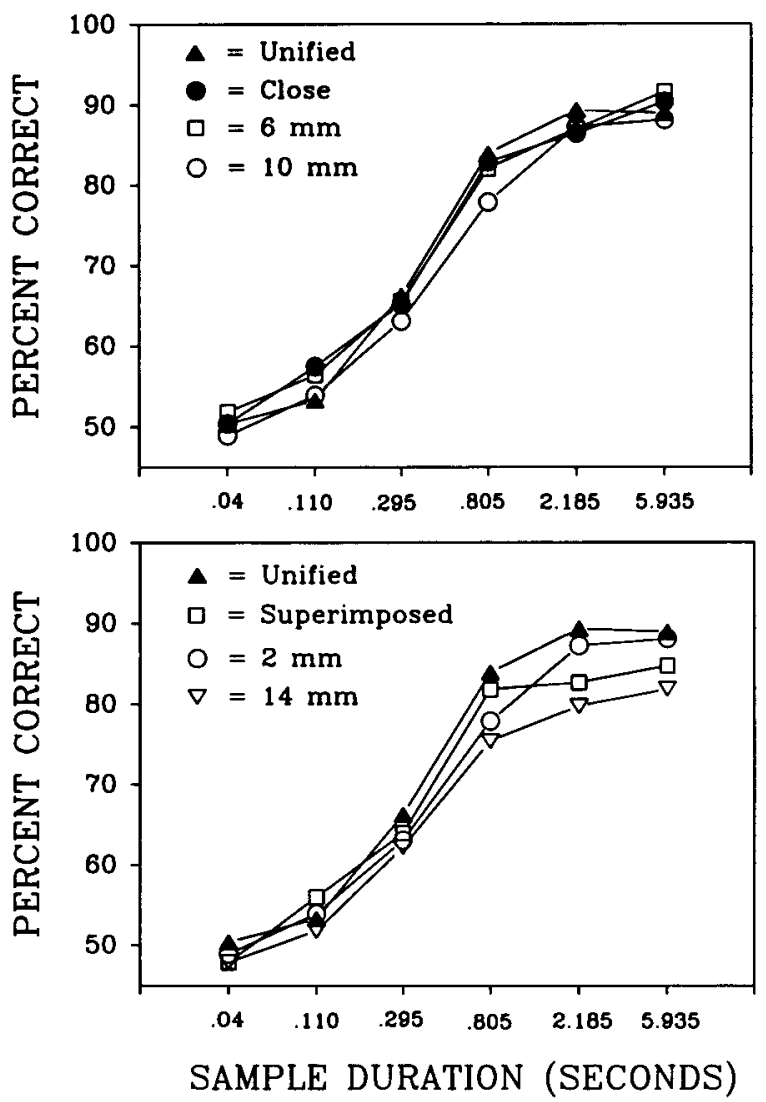

Figure 12. The top panel displays mean percentage correct as a function of sample duration for the unified and three compound conditions found not to be significantly different in Experiment 3. The bottom panel displays the results for the unified (same data as above) and three compound conditions found to be significantly different in Experiment 3. For purposes of comparison, the spacing of the sample duration values is the same as in Lamb and Riley (1981).
Analyses of element properties. These analyses were similar to those conducted for Experiments 1 and 2. To evaluate only asymptotic matching performance, these were limited to results from only the three longest sample durations. The outcomes were similar to those of Experiment 2.

For separated-compound test accuracy, there was a significant interaction between the variable and fixed elements of these compounds and element separation $[F(3,12)$ $=4.8, p<.02]$. As separation increased across the four compounds from $2 \mathrm{~mm}$ to $14 \mathrm{~mm}$, accuracy on tests of the fixed element significantly decreased $[89.9 \%, 85.6 \%$, $79.4 \%, 70.1 \% ; F(3,12)=7.0, p<.01]$, whereas accuracy on tests of the variable element did not significantly change $[84.8 \%, 88.1 \%, 89.4 \%, 87.1 \% ; F(3,12)$ $<1$ ]. Unlike in previous experiments, $14-\mathrm{mm}$ compound accuracy was lower than accuracy with the other separated compounds.

For the proximal compounds there was again a significant interaction between compound type and dimension of test $[F(3,12)=7.2, p<.005]$. Color- and line-test accuracy were not different for the unified compound; line matching was significantly higher for the close, superimposed, and 2-mm compounds.

\section{Discussion}

The most important result of Experiment 3 was that there was no unified compound matching superiority at any sample duration, relative to performance with the close, 6- or 10-mm compounds. Performance on the close compound, for example, was virtually identical to performance on the unified compound at every duration, and when averaged across all durations, it was identical (72.1\%). Despite their larger element separation, accuracy with the 6- and 10-mm compounds was also not different from that with the unified compound.

It seems satisfactory to attribute the differences found between the three remaining compounds and the unified compound to factors unrelated to configural processing. The reason for the lower accuracy with the superimposed and 2-mm compounds has already been discussed. The lower performance with the 14-mm compound seems to be due to the large spatial separation of its elements, perhaps related to a physical limitation in orienting the eyes to two known, but widely separated, sources of information in a limited time period (see Brown, Cook, Lamb, \& Riley, 1984). Whatever the exact source of this limitation, its effects are easily negated by simply bringing the elements slightly closer together (10 mm or less), as indicated by performance with the other separated compounds.

Finally, Experiment 3 resulted in no evidence that the matching of element and compound samples converged toward a common value at long sample durations as predicted by the shared-attention hypothesis. To the contrary, the element and compound conditions appeared, if anything, to be diverging. Subsequent experiments testing sample durations as long as $12 \mathrm{sec}$ also yielded no convergence between the element and compound conditions. This finding suggests that part of the accuracy difference 
between element and compound samples is due to factors unrelated to encoding processes.

\section{GENERAL DISCUSSION}

Three experiments were conducted to assess the hypothesis that pigeons process compound stimuli in different ways, depending on element configuration (Lamb, 1988; Lamb \& Riley, 1981). Using improved stimuli which included careful manipulations of both element spatial separation and configuration, no evidence was found that a "unified" configuration of the color and line elements into a single form conferred any processing advantage over other compound configurations. In comparison with the close compound-dissimilar in physical arrangement, but most comparable in element proximity-the unified compound showed identical levels of overall accuracy in all three experiments. This was true upon the initial transfer to the compound stimuli, under conditions of shared attention, and at all sample durations, short or long. As a consequence, the current results with these better designed stimuli call into serious question Lamb and Riley's (1981) previous evidence for configural compound encoding.

This equivalence between unified and close compound accuracy also suggests that the differential effect of cuing found by Lamb (1988) for "unified" and "separated" compounds may have been due to differences in element separation rather than element configuration. In Lamb's experiments and in the present ones, the asymmetrical, independent, and separate processing of the component elements occurred most strongly with the spatially separated compounds, whereas increasingly symmetrical or shared processing of the elements occurred with the spatially proximal compounds.

The only effect attributable to element configuration reduced rather than increased accuracy. As discussed above, when these small color and line elements are superimposed on one another they appear to be more difficult to process, because of the perceptual masking of the color element by the brighter line element. We have subsequently supported this explanation with experiments in which the reversal of a compound sample's figure/ground brightness relations (to dark line elements surrounding the color element on a white background) have selectively improved color matching in superimposed compounds (Brown, Cook, \& Riley, unpublished data). Lamb and Riley's (1981) superimposed compound was also poorly matched. Both studies thus suggest that the processing of small superimposed compound stimuli involves limiting perceptual factors not present with the larger superimposed compound stimuli produced by in-line projectors (Maki \& Leith, 1973; Maki \& Leuin, 1972).

In the picture of avian compound stimulus processing offered by the present experiments, the principal consideration is the spatial separation of psychologically separate elements. When the elements were close together, processing was shared between the two elements, but as they became more widely separated, processing was increasingly restricted to only one element.

The separated compounds provide the most straightforward evidence for the separate and independent processing of the elements. The competitive and unequal processing of the elements in these compounds suggests that each component was an independent psychological entity. In a number of experiments, spatially separated elements have now produced selective, independent, or restricted processing of one element dimension over the other (the three present experiments; Kraemer \& Roberts, 1985, 1987; Lamb, 1988). Several factors seem to influence which element will be selected. In Experiment 1, the individual birds' preferences determined selection, but with further experience, locational variability appeared to be most important. Kraemer and Roberts $(1985,1987)$ found that the nature of the input modality, and perhaps the patterning of the light source, could also influence this selection.

Although the best evidence for the separate and analytical processing of the elements comes from compounds with physically separated elements, this may not be a necessary condition. Evidence that the proximal compounds were similarly decomposed comes from the successful description of Experiment 1 by the distributed single-element model. A key assumption of such models is that the elements operate as separate and independent units. Hence, the excellent fit of the model to proximal compound performance in Experiment 1 suggests that the elements of the proximal compounds were also being analytically processed at that point in time. The equivalent accuracy observed between the proximal compounds (unified and close) and the physically separated compounds $(6 \mathrm{~mm}$ and $10 \mathrm{~mm}$ ) in Experiments 2 and 3 also suggests that the nature of the element processing in each of these compounds was the same. Interestingly, when placed close together, these "separable" elements appear immune to the selection effects observed in the separated compounds. Lamb's (1988) experiments, and to a more limited extent the present ones, suggest that the shared processing of close elements may be more obligatory (see also Kraemer, Mazmanian, \& Roberts, 1987).

In summary, the results of the present experiments call for a serious reconsideration of our lab's previous evidence for configural compound processing by pigeons (Lamb, 1988; Lamb \& Riley, 1981). These improved experiments, conducted with better designed and unconfounded compound stimuli, examined a wide variety of conditions and found no support for the proposed processing dichotomy in pigeons between a configural, nonanalytic mode and an element-analytic mode as determined by a compound's visual configuration (Riley \& Leith, 1976). Instead, these experiments suggest that pigeons independently process the elements of compounds regard- 
less of their configuration, with the exact form and allocation of this processing strongly controlled by the spatial separation of the elements.

\section{REFERENCES}

Brown, M. F., Cook, R. G., LAMB, M. R., \& RiLeY, D. A. (1984). The relation between response and attentional shifts in pigeon compound matching-to-sample performance. Animal Leaming \& Behavior, 12, 41-49.

Brown, M. F., Morrison, S. K. (1990). Matching to element and compound samples by pigeons: The roles of information load and training history. Journal of Experimental Psychology: Animal Behavior Processes, 16, 185-192.

Ceraso, J. (1985). Unit formation in perception and memory. Psychology of Leaming \& Motivation, 19, 179-220.

FARThing, G. W., Wagner, J. M., Gilmour, S., Waxman, H. M. (1977). Short-term memory and information processing in pigeons. Learning \& Motivation, 8, 520-532.

GARNER, W. R. (1974). The processing of information and structure. Hillsdale, NJ: Erlbaum.

GARNER, W. R. (1976). Interaction of stimulus dimensions in concept and choice processes. Cognitive Psychology, 8, 98-123.

Grant, D. S., MACDonald, S. E. (1986). Matching to element and compound samples in pigeons: The role of sample coding. Journal of Experimental Psychology: Animal Behavior Processes, 12, 160-171.

Kraemer, P. J., Mazmanian, D. S., Roberts, W. A. (1987). Simultaneous processing of visual and spatial stimuli in pigeons. Animal Learning \& Behavior, 15, 417-422.

Kraemer, P. J., RoberTs, W. A. (1985). Short-term memory for simultaneously presented visual and auditory signals in the pigeon. Journal of Experimental Psychology: Animal Behavior Processes, 11, 137-151.

Kraemer, P. J., \& Roberts, W. A. (1987). Restricted processing of simultaneously presented brightness and pattern stimuli in pigeons. Animal Learning \& Behavior, 15, 15-24.

LAMB, M. R. (1988). Selective attention: Effects of cuing on the processing of different types of compound stimuli. Journal of Experimental Psychology: Animal Behavior Processes, 14, 96-104.

LAmB, M. R., \& Riley, D. A. (1981). Effects of element arrangement on the processing of compound stimuli in pigeons (Columba livia). Journal of Experimental Psychology: Animal Behavior Processes, 7, 45-58.

MAKI, W. S., JR., LEITH, C. R. (1973). Shared attention in pigeons. Journal of the Experimental Analysis of Behavior, 19, 345-349.

MAKI, W. S., JR., LEUIN, T. C. (1972). Information processing in pigeons. Science, 176, 535-536.

Miller, R. R., MAruin, N. A., \& BerK, A. M. (1977). Reliability and sources of control of preference for signaled shock. Animal Learming \& Behavior, 5, 303-308.

Rescorla, R. A., \& Cunningham, C. L. (1979). Spatial contiguity facilitates Pavlovian second-order conditioning. Journal of Experimental Psychology: Animal Behavior Processes, 4, 152-161.

RILEY, D. A., \& LEITH, C. R. (1976). Multidimensional psychophysics and selective attention in animals. Psychological Bulletin, 83, 138-160.

Riley, D. A., \& RoItBlat, H. L. (1978). Selective attention and related cognitive processes in pigeons. In S. H. Hulse, H. Fowler, \& W. K. Honig (Eds.), Cognitive processes in animal behavior (pp. 249-276). Hillsdale, NJ: Erlbaum.

SANTI, A., Grossi, V., GiBson, J. (1982). Differences in matchingto-sample performance with element and compound sample stimuli in pigeons. Learning \& Motivation, 13, 240-256.

\section{APPENDIX}

The single-element model of compound processing tested in these experiments assumes that observed accuracy on the compound trial is an additive combination of two types of trials:
(1) trials in which the dimension relevant to the test is processed, and (2) trials in which the relevant test information is not processed. Accuracy for processed trials is estimated from empirical element performance. Accuracy on unprocessed trials is assumed to be at chance $(50 \%)$. Accuracy is then predicted on the basis of the probabilities of whether a particular dimension is processed or not.

Generation of the predicted values using the single-element restriction involved varying only the probability of processing the preferred dimension and fixing the second dimension at 1 probability of processing the first dimension. Hence only three formulae are needed for predicting accuracy when one uses the single element restriction. In this example, the color element is assumed to be the preferred dimension and the term $P(C)$ represents the probability of processing this element. Since accuracy on color and line elements was derived from the empirical data, only the relative probabilities of processing of the preferred dimension are varied in fitting the model.

Color accuracy

$$
\begin{aligned}
= & {[P(\mathrm{C}) \times \text { element color accuracy }] } \\
& +[1-P(\mathrm{C}) \times .5]
\end{aligned}
$$

Line accuracy

$$
\begin{aligned}
= & {[1-P(C) \times \text { element line accuracy }] } \\
& +\{1-[1-P(C)] \times .5\}
\end{aligned}
$$

Overall compound accuracy

$$
=(\text { color accuracy }+ \text { line accuracy }) / 2
$$

A computer program systematically varied this probability of the preferred element by using a least squares criterion between predicted accuracy and observed accuracy to determine the final fit. The predicted values for the single-element model are displayed as diamonds in Figures 3, 5, 6, and 7.

One variation of the model is of additional interest because it places no restriction on the probabilities of processing the two element dimensions. As such, it estimates the total number of processed "elements" required to describe observed compound matching accuracy given actual element performance. This was done by independently varying the probability of processing the second dimension in addition to the first dimension. Simple addition of the terminal values of the two probabilities for each dimension provides an estimate of the number of processed elements needed to describe performance. This value can vary from 0 (no processing of either dimension, leading to $50 \%$ compound accuracy) to 2 (complete processing of both dimensions, leading to $100 \%$ compound accuracy).

It should be noted that this distributed single-element model does not distinguish between whether the allocation of element processing occurs between different trials or within the same trial. Thus, the model is silent as to whether the pigeons exclusively processed the color dimension on $60 \%$ of the trials and on the remaining $40 \%$ of the trials exclusively processed the line dimension, for example, or instead distributed $60 \%$ of their processing capacity to the color dimension and $40 \%$ to the line dimension on every trial. While this is an interesting question in its own right, it does not influence the application of the model as employed in the present article.

(Manuscript received October 16, 1990; revision accepted for publication May 25, 1991.) 